\title{
PARACOCCIDIOIDOMICOSE PULMONAR AGRESSIVA, ENDOTRAQUEITE ESTENOSANTE E COR-PULMONALE SUBAGUDO. DESCRIÇÃO DE UM CASO
}

\author{
E. P. CAMPOS (1), L. N, TORCHIO (2), A. J. M. CATANEO (3) \& L. A. PEROTTI (4)
}

\begin{abstract}
R E S U M O
A paracoccidioidomicose (Pbmicose) atinge os pulmões pela via inalatória, onde se estabelece o complexo primário semelhante ao da tuberculose. A traquéia comprometida pela via tubohemolinfática desenvolveria reação inflamatória em processo granulomatoso levando à obstrução estenosante com asfixia. Acompanhou-se um doente, masculino, 32 anos, branco, natural de Sarutaiá (SP), lavrador, que há 8 meses desenvolveu tosse expectorativa branco-amarelada, diária, sem fatores de melhora ou piora e dispnéia inicial discreta. Há 4 meses, anorexia, fraqueza e astenia. Há 1 mês a dispneia se agravou. Perdeu $15 \mathrm{~kg}$. Tabagista e etilista há 16 anos. Exame físico revelou: PA $-10 / 7 \mathrm{mmHg}$, $\mathrm{FR}=28 \mathrm{bpm}$, peso $31 \mathrm{~kg}$, hipocratismo digital e hipotrofia muscular Tórax enfisematoso e sindrome obstrutivo aos testes de função pulmonar. Coração: P2 desdobrada e hiperfonética. Hepatesplenomegalia. Desenvolveu cor-pulmonale e insuficiência ađrenal à internação, evoluindo após $\mathbf{4 5}$ dias para óbito em insuficiência respiratória aguda asfixiante, apesar da terapia antifúngica ter sido completa. A literatura médica revista não mostrou registro de caso semelhante de cor-pulmonale e insuficiência adrenal de evolução subaguda.
\end{abstract}

UNITERMOS: Paracoccidioidomicose humana - Pulmão - Traquea (Traqueite estenosante) Cor pulmonale sub-agudo

\section{N T R O D U G A O}

A paracoccidioidomicose é doença progressiva crônica que compromete pulmôes, tegumentos, sistema retículo-endotelial e vísceras.

A infecção pulmonar primária acompanhada de disseminação da doença foi demonstrada em modelos experimentais por MACKINNON 11 e RESTREPO ${ }^{13}$.

A existência do complexo de Ghon assim como sua reativação diante de condições de imunodepressão foram relatadas por SEVERO ${ }^{14}$ e SEVERO \& col. ${ }^{15}$.

As vias de penetração bucofaríngea e inalatoria constituem decididamente as principais portas de entrada do fungo $1,2,6,7,9,10,11,13$.

Não se encontrou na literatura descrição de caso de Pbmicose pulmonar agressiva de evolução subaguda com pneumonia cavitária e enfi-

(1) Professor Adjunto - Moléstías Infecciosas e Parasitárias - Faculdede de Medicina - UNESP, Batucatu, Săo Paulo, Brasil

(2) Residente de $1 . \circ$ ano do Moléstias Infecctosas e Parasitárias - Faculdade de Medicina - UNESP

(3) Professor Assistente Doutor - Departamento de Cirurgia - Discplina de Cirurgia Torácica - Faculdade de Medicina - UNESP

(4) Residente de 1.0 ano de Anatomia Patológica - Faculdade de Medicina - UNESP. 
CAMPOS, E. P.; TORChIO, L. N.; CATANEO, A. J. M. \& PEROTTI, L. A. - Paracoccidioidomicose pulmonar agressiva, endotraqueite estenosante e cor-pulmonale subagudo. Descrição de um caso. Rev. Inst. Med. trop. São Paula, 28:185-189, 1986 .

sema evoluindo para endotraqueite granulomatosa obstrutiva e cor-pulmonale.

\section{DESCRIÇAO DO CASO CLINYCO}

J.B., masculino, 32 anos, branco, casado, natural e procedente de Sarutaiá (SP), lavrador (trabalha em lavoura de café, milho e arroz).

Q.D. - Tosse com expectoração há $8 \mathrm{me}$ ses, fraqueza há 4 meses. H.P.H.A. - Há 8 meses tosse com expectoração branco-amarelada, em quantidade moderada, diária, mais intensa à noite, sem fatores desencadeantes e sem fatores de piora ou melhora. De fatores acompanhantes referia apenas discreta falta de ar. Negava febre e hemoptise. Há 4 meses, além dos sintomas referidos, começou a apresentar anorexia, indisposição para o trabalho e fraqueza. Há um mês notou intensificação da dispnéia, não conseguindo caminhar mais de 50 metros. Há 10 dias fez $\mathrm{RX}$ de tórax e exame de escarro no Posto de Saúde de Sarutaiá. senđo de lá encaminhado para este H.C. Refere que perdeu \pm 15 quilos desde $o$ início do quadro.

I.S.D.A. - ardor e queimação ao se alimentar, pois tinha uma fissura na língua. Antecedentes pessoais: fuma desde os 8 anos de idade, há 24 anos portanto, cerca de um maço de cigarro por dia. Bebeu de $1 / 2$ a 1 copo de pinga dos 16 aos 30 anos de iđađe, há 2 anos não bebe. Sempre morou em Sarutaia, nunca visitou outras áreas do Brasil. Negava contato com tuberculose. Antecedentes familiares: sem interesse para o caso.

Exame Físico Geral: PA $=10 \times 7 \mathrm{mmHg}$; $\mathrm{P}=\mathrm{FC}=80 \mathrm{bpm} ; \mathrm{FR}=28 \mathrm{mpm} ;$ peso $=31 \mathrm{~kg}$. Paciente em REG, caquético, hidratado. Mucosas descoradas ++ , edema: ausente; extremidades: hipocratismo digital. Exame físico especial: dentes em mau estado de conservação. Torax tipo enfisematoso: FTV aumentado nos campos médios, bilateral, elasticidade e expansibilidade torácicas diminuídas globalmente. Presença de submacicez nos campos pulmonares médios, hipersonoridade nas bases. O restante som claro pulmonar. MV presente e diminuido globalmente, presença de estertores subcrepitantes nos dois pulmões. Bulhas ritmicas hiperfonéticas com desdobramento de P2. Fígado palpável $a \pm 4 \mathrm{~cm}$ do RCD na LHCD e à $\pm 8 \mathrm{~cm}$ do apên- dice xifoide, características normais, indolor; baço percutível e palpável há $\pm 3 \mathrm{~cm}$ do $\mathrm{RCE}$, indolor, pouco endurecido. Exame neurológico normal. Hipóteses diagnósticas: Desnutrição protéico-calórica. Enfisema pulmonar. Paracoccidioidomicose pulmonar? Tuberculose pulmonar?

Conduta: internação para investigação clínico-laboratorial, seguida de terapia pela anfotericina $\mathbf{B}$.

\section{Exames Subsidiários}

24/11/83 - Hemograma: $\mathrm{Ht}=39 \% ; \mathrm{Hb}=11,2$ $\mathrm{g} \% ; \mathrm{GB}=21000 \mathrm{~mm}^{3}$

$25 / 11 / 83-$ Uréia $=16 \mathrm{mg} \%$; Creatinina $=0,8$ $\mathrm{mg} \%$.

01/12/83 - Hemograma: Ht $=38 \% ; \mathbf{H b}=9,9$ $\mathrm{g} \%$; VHS $=41 \mathrm{~mm}$ 1a. hora; GB $=16.600$; contagem diferencial: bastão $=8 \%$; segmentado $=73 \%$; basófilos $=2 \%$; linfócito $=$ $10 \%$; monócito $=7 \%$. OBS: neutrófilos com moderada granulaçăo tóxica e $1 \%$ de linfócito atípico.

Bioquímicos: $\mathrm{Na}=135 \mathrm{mEq} / 1 ; \mathrm{K}=4,7 \mathrm{mEq} / 1$; $\mathrm{Ca}=9,0 \mathrm{mEq} / 1$

Fosfatase alcalina $=378 \mathrm{U}(15-69 \mathrm{~N})$

$\mathrm{BD}=0,06 \mathrm{mg} \% ; \mathrm{BI}=0,69 \mathrm{mg} \% ; \mathrm{TGO}=60 \mathrm{U} ;$ $\mathrm{TGP}=68 \mathrm{U}$.

Em 03/12/83 chegaram os resultados dos exames citopatológicos de escarro que revelar ram em 3 amostras pesquisadas, grande quantidade de Paracoccidioides brasiliensis.

Os exames de escarro para pesquisa de BK foram todos negativos -4 amostras.

\section{Radiologia Torácica}

Partes moles e óssea - nada digno de nota. Campos pulmonares: enfisema de bases com retificação das cúpulas diafragmáticas; mediastino: desviado para a esquerda, sinal da silhueta cardíaca e ângulo de carina modificado, traquéia: discreta diminuição da luz, lesão apical cavitária direita, exsudatos intersticiais flococotonosos fibrolineares bilaterais, bronquiectasias prováveis de lobo inferior direito e de lobo médio. Evolução radiológica: reação enđotraqueal com diminuição da luz, fibrose pulmonar 
CAMPOS, E. P.; TORChIO, L. N.; CATANEO, A. J. M. \& PEROTTI, L. A. - Paracoccldioidomicose puimonar agressíva, endotraqueite estenosante e cor-pulmonale subagudo. Descrição de um caso. Rev. Inst. Med. trop. Sāo Paulo, 28:185-189, 1986.

no tinal da terapia e sinais indiretos de hipertensao.

\section{Evolução}

Na primeira semana de fevereiro, o paciente apresentou crise de insuficiència respiracorıa aguda por obstrução alta que cursou para o êxito letal.

\section{Éxame Anátomo patológico}

Macroscopia - os pulmões exibiram intensa fibrose de localização perihilar, estendendose de maneira homogênea do campo pulmonar médio até a pleura. Os ápices mostraram mûltiplas cavitaçóes bronquiectásicas e enfisema sub-pleural. Os lobos inferiores continham numerosas condensaçōes puntiformes, brancacen. tas confluentes de distribuição miliar. A traquéia exibiu mucosa de aspecto granuloso $e$ intensa estenose cicatricial proximal com redução da luz. Notou-se moderada quantidade de secreção catarral na luz traqueabrônquica. $\mathrm{O}$ corpulmonale foi documentado pela hipertrofia ventricular đireita e pela congestão passiva crônica do fígado. Os linfonodos do hilo pulmonar e a supra-renal esquerda apresentaram lesöes nodulares brancacentas necróticas.

Microscopia - a histologia dos pulmões demonstrou fibrose com formação de cavidades bronquiectásicas parcialmente epitelizađas, granulomas confluentes contendo numerosas células gigantes com P. brasiliensis, fragmentados ou integros. Observaram-se focos broncopneumónicos e traqueobronquite crônica catarral com metaplasia escamosa. Inflamação granulomatosa paracoccidióidica com destruicăo parcial dos anéis cartilaginosos e intensa cicatrizacão fibrótica determinaram estenose da luz tra. queal. Os linfonodos do hilo pulmonar e supra-renal esquerda exibiram extensa necrose, margeada, por granulomas contendo fungos fragmentados.

\section{COMENTARIOS}

A Pbmicose pulmonar parece ter história natural semelhante à da tuberculose, de acordo com as observaçōes descritas por FIALFO', LIMA $^{9}$ e outros 2,14 , podendo ser primária benig na, progressiva aguda ou crônica e disseminada, segundo LONDERO \& col. ${ }^{10}$. A forma progressiva conduziria ao agravamento da lesão pulmonar e a disseminação para os diversos órgãos.

Formas graves disseminadas da Pbmicose foram assinaladas nas fases crónicas e de inoculação traumática do fungo $4,6,9,10,14,15$.

O presente caso desenvolveu pneumonia progressiva tipo agressiva, caracterizada por enfisema, bronquiectasias, cavitação, miliarização, endotraqueite e hipertensão pulmonar seguida de cor-pulmonale.

O enfisema observado na Pbniticose parece depender de dois fatores: da própria doença e do tabagismo. Este ültimo facilitaria a progressão da lesão pulmonar preexistente produzida pela Pbmicose 1,2,4,6,8,9.

Bronquiectasias e cavitação seriam oriundas da atividade da doença no aparelho respiratório. A miliarização configuraria disseminação e dōença paracoccidioidomicótica ativa. Formas cavitárias e miliarizadas sugeririam doentes debilitados $2,4,6,7,9,10,14$.

A hipertensão pulmonar precoce verificada há 4 meses do início da doença paracoccidioidomicótica chamou-nos atenção. Após 20 dias da internação configurou-se cor-pulmonale cli. nico com " $p$ " pulmonale ao traçado eletrocardiográfico. O cor-pulmonale na Pbmicose parece ser descrito habitualmente na fase crônica da doença como assinalaram alguns Autores $1,4,8$.

f preciso salientar esse fato não habitual que denunciou a grave lesão pulmonar existente precocemente acompanhada de cor-pulmonale.

A endotraqueíte inicialmente discreta à internação evoluiu para obstrução total das vias aéreas superiores conduzindo o paciente ao êxito letal por insuficiência respiratória asfixiante apesar do exame otorrinolaringológico ter sido normal a internação. A gravidade da endotraqueite granulomatosa se deveu a disseminação observada no presente caso durante sua evolução fatal. Reação endotraqueal foi descrita à internação de um doente por MONTESSI \& col. ${ }^{12}$. A endoscopia mostrou massa tumoral cuja biopsia revelou granuloma paracoccidioidomicótico. 
CAMPOS, E. P.; TORChIO, L. N.; CATANEO, A. J. M. \& PEROTTI, L. A. - Paracoccidioidomicose pulmonar agressiva, endotraqueite estenosante e cor-pulmonale subagudo. Descrição de um caso. Rev. Inst. Med. trop. São Paulo. 28:185.189, 1986.

A hepatesplenomegalia descrita ao exame clínico assim como o comprometimento adrenal evidenciado ao anátomo patológico seriam explicados pela disseminação precoce da Pbmicose observada no doente. O envolvimento precoce da supra-renal em doentes de Pbmicose, fora da forma crônica, foi sugerido por CAMPOS \& col. ${ }^{4}$. A lesão anatomopatológica inicial da supra-renal estaria na dependência da Pbmi. cose pulmonar progressiva assinalada no doente 3,4 , visto que essa glândula é atingida mais comumente em doentes com micose de Lutz de longa duração $2,4,5,6,7$.

O uso de corticóide em dose baixa contribuiu para acentuar a imunodepressão assim como justificar a hipopotassemia assinalada durante esse período no paciente 4,16 . A infusão de Anfotericina $\mathrm{B}$ associada ao corticóide acen. tuaria a hipocalemia possivelmente por sinergismo de ação espoliativa sobre esse elemento quimico, segundo sugeriram TREZZA \& col. ${ }^{16}$.

Nosso caso clínico poderia ser esquematicamente apresentado da seguinte maneira:

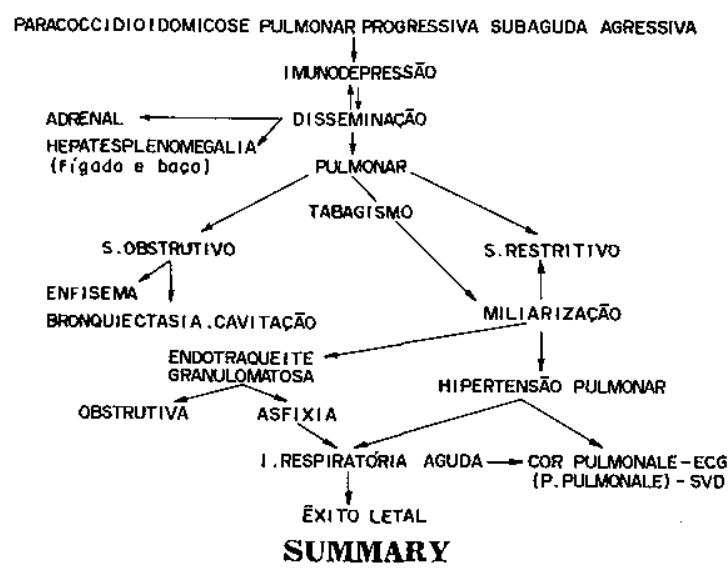

Aggressive pulmonary paracoccidioidomycosis, stenosante tracheitis and subacute Cor pulmo. nale. Report of a case.

Paracoccidioidomycosis (Pbmycosis) reaches the lungs through the respiratory tract where it initiates the primary infection. Although the disease produces a variety of extra. pulmonary manifestations, the lungs are involv. ed in a large number of cases. The trachea is affected by Pbmycosis lymphohematogenic dissemination causing a granulomatous process leading to airway obstruction and secondary asphyxia. A 32 years old male, farm worker coming from Sarutaia (S.P.) was studied in our hospital. The patient has developed, since 8 months ago a white productive daily cough and a mild dyspnea. Four months later, the mentioned symptoms worsened and anorexia and asthenia appeared. One month previously to hospital admission the dyspnea increased and a weight loss of $15 \mathrm{~kg}$ was observed. He has been a heavy smoker and a heavy drinker during the last 16 years. On physical examination it was observed: blood pressure $=100 / 70$ $\mathrm{mm} / \mathrm{Hg}$, respiratory rate $=28 \mathrm{pm}$, body weight $=31 \mathrm{Kg}$. Pulmonary emphysema, clubbing, loud an splitting $\mathbf{S 2}$, right ventricular hypertrophy on E.K.G., hepatosplenomegaly and signs of obstructive pulmonary disease allowed the diagnosis of a "Cor pulmonale" - like syndrome. Amphotericin $B$ was administred at the dose of $1 \mathrm{mg} / \mathrm{Kg}$ body weight. A relative adrenal insufficienoy was observed early during the treatment. 45 days after hospitalization the patient developed an acute respiratory insufficiency caused by tracheal obstruction and aggravated Cor pulmonale. In spite of intensive care he died. The tracheal stenosis and sub acute Cor pulmonale reported in this patient is a rare ma nifestation of human pulmonary pbmycosis.

\section{REFERENCTAS BIBLYOGRAFICAS}

1. AFONSO, J. E.; NERY, L. E.; ROMALDINI, H.; BOGOSSIAN, M. \& RIBEIRO-RATTO, O. - Funçáo pul. monar na paracoccidioidomicose (blastomicose sul-americana). Rev. Inst. Med. trop. S. Paulo, 21: 269-280, 1979 .

2. BRASS, K. - Observaciones sobre la anatomia patologica, patogenesis $y$ evolucion de la paracoccidoldomicosis, Mycophatologia (Den Haag), 37: 119.138, 1969.

3. CAMPOS, E. P.; CARNEIRO, A. C.; RAMOS, M. D.: MENDES, R. P. \& MEIrA, D. A. - Avaliação da função supra-renal na vigêncta do tratamento da blastomicose sul-americana pela Anfotericina $B$. In: JORNADA CIENTIFTCA DA FACULDADE DE CIENCIAS MEDICAS E BIOLOGICAS DE BOTUCATU, 5., Botucatu, 1975. p. 74.

4. CAMPOS, E. P.; SARTOR, J. M.; HECHT, M. L. \& FraNCO, M. F. - Aspectos clinicos e evolutivos de 47 doentes tratados pela Anfotericina B. Rev. Inst Med. trop. S. Paulo, 26: 212-216, 1984.

5. DEL NEGRO, G. - Localizaçăo supra-reñu da blastomicose sul-americana. São Paulo, Saraiva, 1961. (Tese de livre-docência - Faculdade de Medicina da Uni versidade de São Paulo). 
CAMPOS, E. P.; TORChIO, L. N.; CATANEJ, A. J. M. \& PEROTTI, L. A. - Paracoccidioidomicose pulmonar agressiva, endotraqueite estenosante e cor-pulmonale subagudo. Descriçăo de um caso. Rev. Inst. Med. trop. São Paulo, 28:185-189, 1986 .

6. FIALKO, A. S. - Locallzaçōes pulmonares da "Micose de Lutz". Anatomia patológica e patogenia. Importância de seu estudo na patologia pulmonar. Rio de Janeiro, 1946. (Tese).

7. FRANCO, M. F. \& MONTENEGRO, M. R. G. - Anatomia patológica. In; DEL NEGRO, G.; LACAZ, C. S. \& FIORILLO, A. M. - Paracoccidioidomicose. Blastomicose sul-americana. S巨̋o Paulo, Sarvier: EDUSP, 1982. cap. 9, p. 97-115.

8. LEMLE, A.; WANKE, B.; MIRANDA, J. L.; KROPF, G. L.; MANDEL, M. B. \& MANDEL, S. - Pulmonary function in paracoccidioidomycosis (South American Blastomycosis). An analysis of the obstructive defect. Chest, 83: 827.828, 1983.

9. LIMA, F. X. P. - Contribulção ao eqtudo clínleo e radiológico da blastomicose pulmonar. São Paulo, 1952. (Tese de doutoramento - Faculdade de Medicina da Universidade de São Paulo).

10. LONDERO, A. R.; RAMOS, C. D. \& LOPES, J. O. - Paracoccidioidomicose. Clessificaçăo das formas clí. nicas, Rev, urug. Pat. clín, microblol., 14: 3-9, 1976.

11. MaCkinoNn, J. E. - Pathogenesis of South American Blastomycosis. Trans. roy. Soc, trop. Med. Hyg., 53: 484-494, 1959.
12. MONTESSI, A.; SOUZA, L. R. G. L.; GUTMARAES, A.; JANINI, M. C. R. \& GUEDES E SILVA, J. B. Paracoccidioidomicose endobronquica. Apresentação do um caso. XXI Congresso Brasileiro de Pneumologla, São Paulo. J. Pneumol. (Supl.): 198, 1982.

13. RESTREPO, M. A.; GREER, D. L. \& VASCONCELLOS, M. - Paracoccidioidomycosis - a review. Rev, med. Vet. Mycol., 8: 97-123, 1973.

14. SEVERO, L. C. - Paracoccidioidomicose. Estudo clinico e parasitológico das lesōes pulmonares e seu diaznóstico. Porto Alegre, 1979. (Dissertaçăo de mestrado - Universidade Federal do Rio Grande do Sul).

15. SEVERO, L. C.; PALOMBINI, B. C.; UTZ, E. \& BRAUN, S. M. - Paracoccidioidomicose pulmonar resultante da reativaçăo de foco quiescento em paciente imunosuprimido. J. Pneumol., 6: 21-22, 1980.

16. TREZZA, E.; DILLON, N. L. \& CAMPOS, E. P. Alteraçōes eletrocardiográficas durante o uso de Anfotericina B. Congresso Brasileiro do Cardiologia, Curltiba, 1972. p. 28 .

Recebido paræ publicação em 2/7/1985. 